University of Wollongong

Research Online

Faculty of Engineering - Papers (Archive)

Faculty of Engineering and Information

Sciences

2007

\title{
Out-of-field dose equivalents delivered by proton therapy of prostate cancer
}

\author{
A. Wroe \\ University of Wollongong, awroe@uow.edu.au \\ Anatoly B. Rosenfeld \\ University of Wollongong, anatoly@uow.edu.au \\ R. Schulte \\ Loma Linda University Medical Center, California
}

Follow this and additional works at: https://ro.uow.edu.au/engpapers

Part of the Engineering Commons

https://ro.uow.edu.au/engpapers/464

\section{Recommended Citation}

Wroe, A.; Rosenfeld, Anatoly B.; and Schulte, R.: Out-of-field dose equivalents delivered by proton therapy of prostate cancer 2007.

https://ro.uow.edu.au/engpapers/464

Research Online is the open access institutional repository for the University of Wollongong. For further information contact the UOW Library: research-pubs@uow.edu.au 


\title{
Out-of-field dose equivalents delivered by proton therapy of prostate cancer
}

\author{
Andrew Wroe ${ }^{\text {a) }}$ and Anatoly Rosenfeld \\ Centre for Medical Radiation Physics, University of Wollongong, New South Wales, Australia \\ Reinhard Schulte \\ Department of Radiation Therapy, Loma Linda University Medical Center, Loma Linda, California 92354
}

(Received 11 April 2007; revised 24 June 2007; accepted for publication 26 June 2007; published 7 August 2007)

\begin{abstract}
Measurements were performed to assess the dose equivalent outside a primary proton treatment field, using a silicon-on-insulator (SOI) microdosimeter. The SOI microdosimeter was placed on the surface of an anthropomorphic phantom and dose equivalents were determined as a function of lateral distance from a typical passively scattered and modulated prostate treatment field. Measurements were also completed within a polystyrene plate phantom as a function of depth for a distance of $5 \mathrm{~cm}$ from the field edge, as function of lateral distance from field edge at two different depths, and as a function of distance from the distal edge on the central beam axis. The dose equivalent at the surface of the anthropomorphic phantom decreases from 3.9 to $0.18 \mathrm{mSv} / \mathrm{Gy}$ when the lateral distance from the proton field edge increases from 2.5 to $60 \mathrm{~cm}$. Measurements along the proton depth dose distribution at a constant distance of $5 \mathrm{~cm}$ from the primary field edge indicate a decrease in dose equivalent as a function of depth, with a $38 \%$ decrease relative to the surface dose at a depth of $5 \mathrm{~cm}$ in polystyrene. Measurements completed as a function of lateral distance from the primary field at two separate depths within polystyrene illustrate a convergence of the dose equivalent at approximately $20 \mathrm{~cm}$ from the primary field edge. Past the distal edge of the spreadout Bragg peak dose equivalents decrease exponentially for increasing distance, with an initial value of $1.6 \mathrm{mSv} / \mathrm{Gy}$ at $0.6 \mathrm{~cm}$ from the distal edge. Silicon microdosimetry measurements were also compared with published results obtained utilizing different measurement techniques. This study demonstrates the applicability of SOI microdosimetry in determining the dose equivalent outside proton treatment fields, and provides valuable information on the dose equivalent both at the surface and at depth experienced by prostate cancer patients treated with protons. (C) 2007 American Association of Physicists in Medicine. [DOI: 10.1118/1.2759839]
\end{abstract}

Key words: proton therapy, dose equivalent, neutrons, microdosimetry

\section{INTRODUCTION}

Presently one of the major health risks mankind faces is cancer. Projections are that one in three people will suffer from this disease or side effects of its treatment at one stage in their life. ${ }^{1}$ Because of the deleterious effects that cancer and often current treatment forms are having on the human population, better treatment techniques are constantly being sought. Besides surgery, external beam radiation therapy is a mainstay of cancer treatment and cure. Over the last two decades highly conformal radiation therapy techniques have been developed, including intensity modulated photon radiotherapy (IMRT) and radiotherapy utilizing protons and carbon ions. One drawback of these techniques is that they may increase the contribution of lower doses to large volumes of normal tissues outside the target volume. ${ }^{2}$

High energy protons are an important innovation in external beam radiation therapy, providing highly conformal dose distributions, thus sparing normal tissues through the benefits afforded by the Bragg peak. These dose distributions provide a smaller integral dose to surrounding normal tissues when compared with conventional x-ray treatments. Despite the dose sparing properties of protons and heavy ions, they do have the potential to produce unwanted dose outside the primary field due to secondary particles such as neutrons through inelastic nuclear interactions with the beam modifying devices and tissues traversed. This is of concern as neutrons are known to be more effective than photons and high energy protons at producing radiation induced cancers. ${ }^{2}$ The primary beam is also scattered as it traverses the patient, contributing additional unwanted dose outside the primary treatment field.

The production of secondary particles with therapeutic proton beams and the delivery of unwanted dose equivalent outside the treatment field is closely related to the delivery technique employed. ${ }^{2}$ Proton delivery techniques can be categorized as passive or active in the delivery of a uniform dose to the treatment volume. Passive techniques, which have been most commonly used in the clinical setting, including prostate cancer treatment, ${ }^{3,4}$ spread the beam laterally using a combination of gold and Lexan foils ${ }^{5}$ and in depth by using a rotating plastic wheel. ${ }^{6}$ The beam is then collimated by brass or Cerrobend ${ }^{\circledR}$ apertures and its penetration depth is varied by means of a wax bolus. Active techniques, ${ }^{7-10}$ currently only practiced in a clinical situation at the Paul Scherrer Institute (PSI), employ a magnetically 


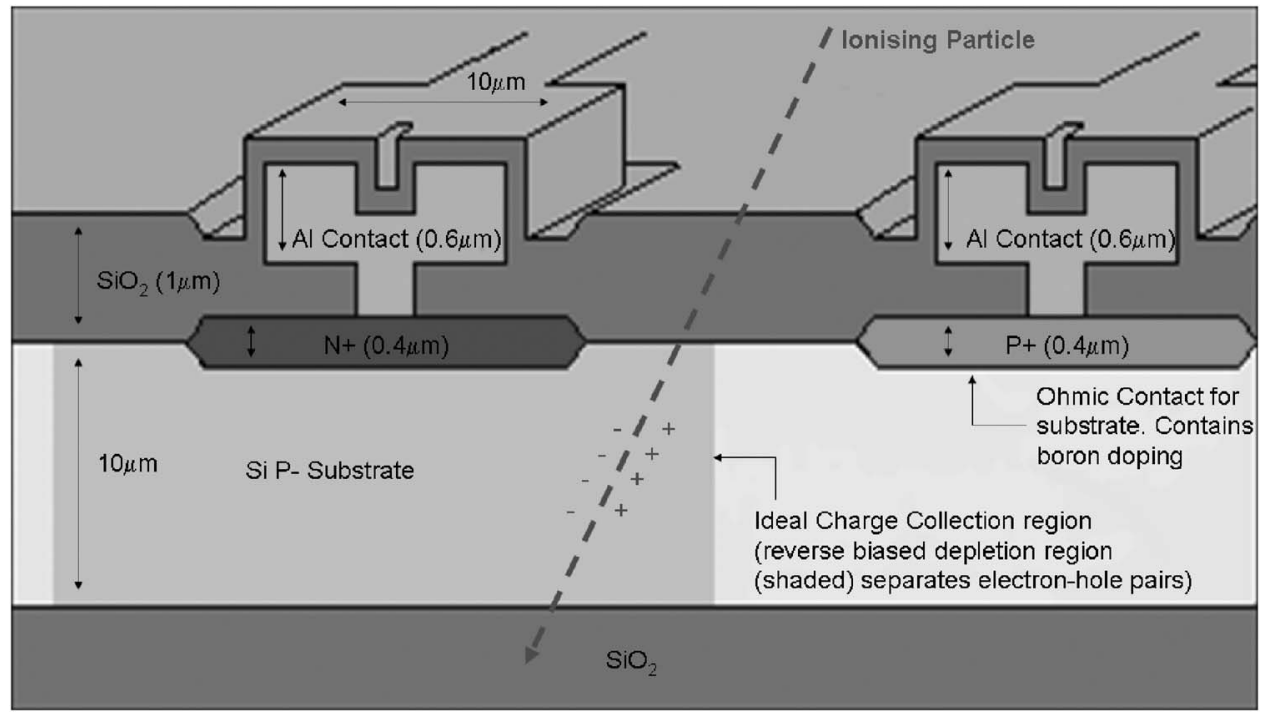

FIG. 1. Basic SOI diode array structure of the microdosimeter developed at the CMRP, University of Wollongong. guided proton pencil beam in combination with dynamic changes of beam energy and beam intensity during treatment. Comparatively, passive beam delivery techniques provide a larger source of secondary neutrons due to the additional presence of scattering foils, modulator, aperture, and bolus.

Measurements of out-of-field doses delivered in both active and passive clinical proton modalities are scarce ${ }^{11-13}$ and have primarily concentrated on the measurement of neutrons with Bonner spheres and bubble detectors. Additional measurements are required and should be performed systematically for different beam delivery techniques utilizing the same method of measurement. We have approached this problem utilizing silicon-based microdosimetry, which is characterized by a superior spatial resolution and directly provides information on the dose equivalent using established quality factors. ${ }^{14}$ The measurements reported here provide information on the depth and lateral distance dependence of the dose equivalent for a passively scattered proton beam used in a typical prostate cancer treatment. Silicon microdosimetry measurements were compared with published results obtained utilizing different measurement techniques.

\section{EXPERIMENTAL METHOD}

This study utilized a solid-state microdosimeter with silicon-on-insulator (SOI) technology as illustrated in Fig. 1. The large size of tissue equivalent proportional counters (TEPC), classically used in microdosimetry measurements, ${ }^{15}$ made it unsuitable for measurements in small increments close to the primary proton treatment field edge and within a phantom structure. The SOI microdosimeter, presented in detail in Ref. 16, comprised an array of 4800 silicon cells, each with a physical size of $30 \times 30 \mu \mathrm{m}$, on a single chip with a sensitive volume (SV) thickness of $10 \mu \mathrm{m}$. This small size allowed for accurate placement within the phantom structure and close to the primary field edge. The application of SOI microdosimeters in therapeutic neutron radiation fields has been studied in detail, and good agreement was observed between the derived microdosimetry spectra of both the SOI and TEPC devices. ${ }^{17,18}$ This device has also been used extensively in hadron and proton therapy, particularly in primary beam microdosimetry measurements. $16,17,19-21$

In this experimental setup, the SOI microdosimeter, preamplifier, and accompanying electronics were situated within an aluminum probe assembly to allow for a low noise environment to be achieved. The wall thickness of the probe was $900 \mu \mathrm{m}$, with a $4 \mu \mathrm{m}$ aluminum window immediately in front of the microdosimeter to allow for the transport of low range secondaries into the microdosimeter. The microdosimeter probe was housed within a Perspex probe holder to enable accurate placement throughout the experiment. This setup is described further in Ref. 21. A $0.5 \mathrm{~mm}$ thick polyethylene converter affixed immediately anterior to the SOI microdosimeter allowed for the conversion of neutrons to recoil protons that could then be detected within the SV. Such converters have been employed in previous neutron and mixed field measurements with this device. ${ }^{17,20}$

All measurements were completed using a patient specific aperture-bolus combination, and proton energy of $225 \mathrm{MeV}$ to simulate a typical radiation field delivered in a clinical prostate case. The maximum aperture diameter was $8 \mathrm{~cm}$ both in lateral and vertical direction. Additional measurements were also completed in some cases using a $13 \mathrm{~cm}$ diameter circular aperture (quality assurance or QA aperture) with no bolus present to demonstrate the effect of aperture size and bolus on the dose equivalent. To conduct a thorough study of the dose equivalent delivered outside the treatment field, four separate experimental configurations were used, each measuring a different aspect of the radiation field. In the first case, the patient was simulated with an anthropomorphic phantom (Rando ${ }^{\circledR}$ Phantom, The Phantom Laboratory, Salem, NY) housed within a regular patient immobilization system (Fig. 2). The microdosimeter (MD) was placed on the phantom surface at the height of the central axis, and microdosimetry measurements were performed to determine the 


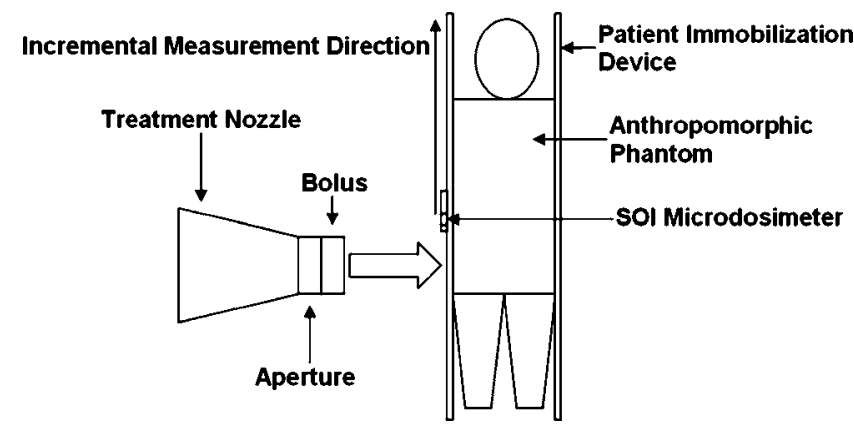

FIG. 2. Experimental setup for surface measurements as a function of distance from the field edge.

change in dose equivalent as a function of increasing lateral distance from the field edge.

Additional measurements were conducted with the anthropomorphic phantom replaced with a stack of polystyrene blocks. Using this experimental setup, three measurement series were completed (Fig. 3). First, the MD was placed at different depths within the polystyrene phantom at a distance of $5 \mathrm{~cm}$ from the field edge to estimate the change in dose equivalent as a function of depth within the patient (series M1). Measurements were also completed to assess the change in dose equivalent as a function of lateral distance from the field edge at different depths within the polystyrene phantom (series M2). Finally, measurements were completed to assess the dose equivalent beyond the distal edge of the spread-out Bragg peak (SOBP) (series M3), with the distal edge being defined as the point where the primary dose reached $50 \%$. In all cases, dose equivalent was normalized to the proton dose delivered at isocenter.

\section{II.A. Determination of dose equivalent}

The dose equivalent $(H)$ was defined in Ref. 14 to characterize the effects of low doses of ionizing radiation on health. It is defined by the ICRU as

$$
H=Q D,
$$

where $Q$ is the average quality factor of the radiation field and $D$ is absorbed dose in tissue at a given point of interest. The unit of dose equivalent is the Sievert (Sv), with $Q$ specified by ICRU Report $40 .^{22}$

In this study, the method outlined in Refs. 14 and 15 was used in the determination of $H$ with specified $Q$ values, which are dependent on lineal energy. The conversion of measured microdosimetry spectra (Fig. 4) to dose equivalent

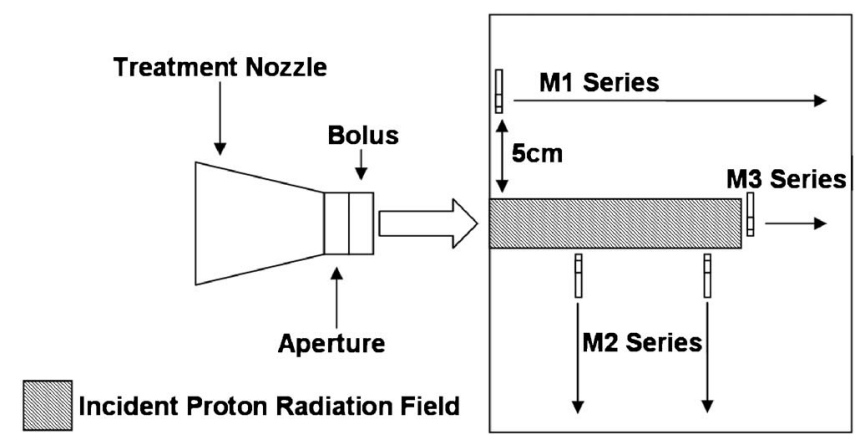

FIG. 3. Experimental setup for experimental measurements within a polystyrene phantom. Note the three separate measurement series conducted with this experimental setup. M1 determined the change in dose equivalent as a function of depth; M2 provided the change in dose equivalent as a function of distance from the field edge within the polystyrene phantom; and M3 indicated the change in dose equivalent past the distal edge.

proceeded in two steps: (1) calculation of absorbed dose to tissue at each point of measurement; (2) calculation of the dose-averaged quality factor at that point.

Microdosimetric data were acquired as energy deposition spectra $f(E)$ vs $E$, where $f(E)$ is the relative frequency of events and $E$ the energy determined from the charge collected within in a single silicon SV. Energy deposition events were corrected for 0.8 charge collection efficiency ${ }^{23}$ and then used to calculate the absorbed dose to silicon by integrating over the spectrum [Eq. (2)],

$$
D_{\mathrm{Si}}=\frac{\int_{0}^{\infty} f(E) E d E}{\rho_{\mathrm{Si}} V n_{\text {cells }}},
$$

where $n_{\text {cells }}$ is the number of SVs in a given array used (4800), ${ }^{16} V$ is the volume of a $30 \times 30 \times 10 \mu \mathrm{m}^{3}$ individual detection element, and $\rho_{\mathrm{Si}}$ the density of silicon. ${ }^{24}$ The tissueequivalent (TE) dose $D_{\mathrm{TE}}$ can then be determined by using the ratio of total stopping powers of protons for silicon, $S_{\mathrm{Si}}$ and tissue $S_{\mathrm{TE}}$ (Ref. 25) (referred to in this document as TE conversion factor $\zeta$ ),

$$
\frac{D_{\mathrm{Si}}}{D_{\mathrm{TE}}}=\frac{S_{\mathrm{Si}}}{S_{\mathrm{TE}}}=\zeta .
$$

From previous research ${ }^{16,17,19}$ and comparisons with TEPCs, the most appropriate value of $\zeta$ was determined to be $\zeta$ $=0.63$, which was used in this work.

The first step in calculating the fraction of the doseaverage quality factor is to convert the $f(E)$ spectra into a lineal energy spectra using the average chord length $\langle l\rangle$,
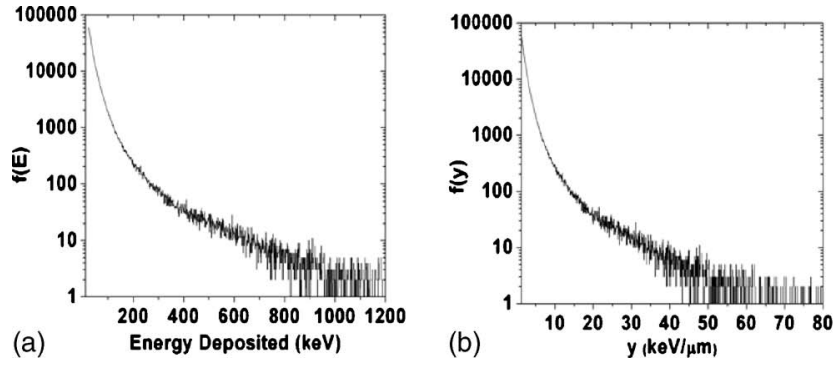

Medical Physics, Vol. 34, No. 9, September 2007

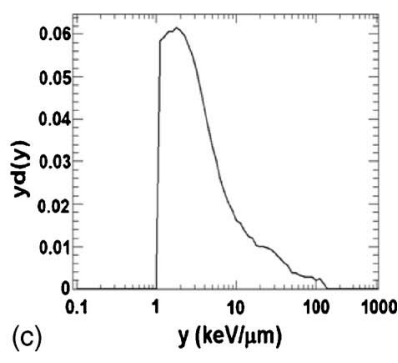

FIG. 4. Examples of distributions obtained using the SOI microdosimeter including (a) $f(E)$ vs $E$ distribution; (b) $f(y)$ vs $y$ distribution; (c) $y d(y)$ vs $y$ distribution. 


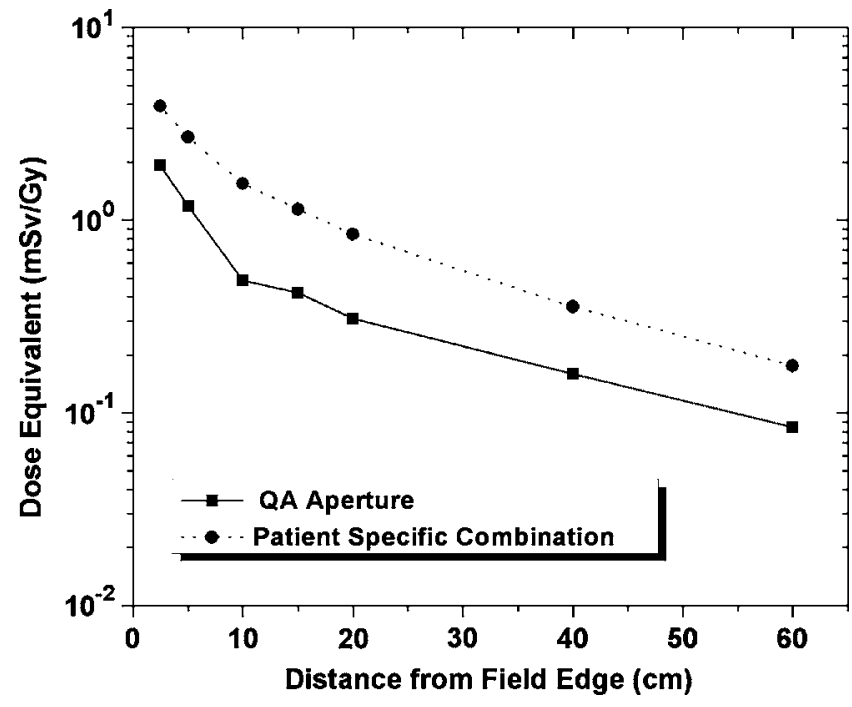

FIG. 5. Results as measured with the SOI microdosimeter for both the patient specific aperture/bolus portal and the $13 \mathrm{~cm}$ circular QA aperture with no bolus present.

$$
\langle l\rangle=\frac{4 V}{A \zeta},
$$

where $V$ is the volume and $A$ the surface area of the SV. For the $30 \times 30 \times 10 \mu \mathrm{m}^{3}$ sensitive volume the average chord length is $19.05 \mu \mathrm{m}$. Note the use of the TE conversion factor to calculate the average chord length in tissue. Dividing $E$ by $\langle l\rangle$ in the energy deposition spectra provides the lineal energy spectra. The normalized dose-weighted lineal energy distribution is given by

$$
d(y)=\frac{y f(y)}{y_{f}},
$$

where

$$
y_{f}=\int_{0}^{\infty} y f(y) d y
$$

is the frequency averaged lineal energy. Note that $d(y)$ gives the fraction of total absorbed dose in the interval $[y, y+d y]$.

In order to determine dose equivalent, a $y$-dependent quality factor $Q(y)$ was introduced as prescribed by ICRU Report $40 .^{22}$ From the $d(y)$ distribution, the dose equivalent at that point in space can be determined using Eq. (7),

$$
H=D_{\mathrm{TE}} \int_{0}^{\infty} Q(y) d(y) d y=Q_{\mathrm{avg}} D_{\mathrm{TE}},
$$

where $Q_{\text {avg }}$ is the average quality factor.

\section{RESULTS}

Figure 5 presents the results of dose equivalent values on the surface of the anthropomorphic phantom as a function of distance from the incident proton field edge. It is evident from these results that the aperture and bolus used within a typical prostate cancer patient treatment increased the dose equivalent by a factor of 2-4 when compared to the $13 \mathrm{~cm}$

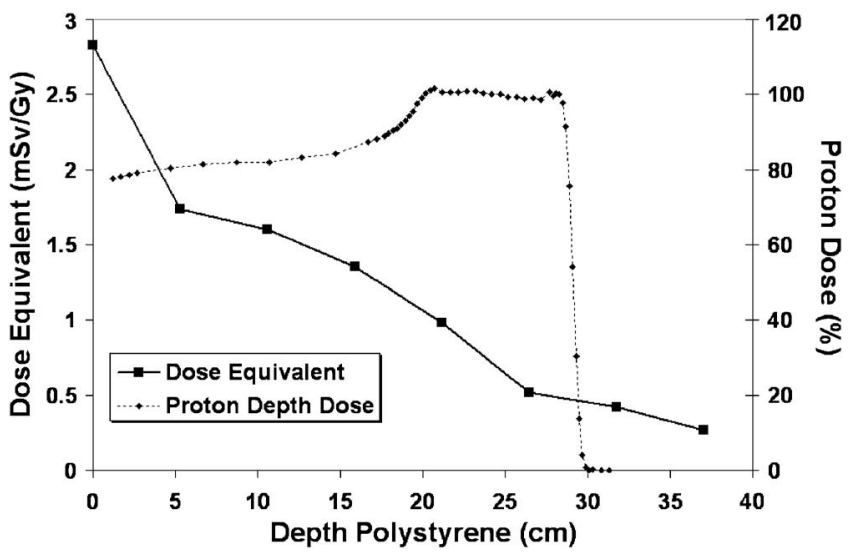

FIG. 6. Dose equivalent at $5 \mathrm{~cm}$ from the field edge compared with the depth dose distribution of the proton beam as measured along the central axis with a Markus chamber. The proton dose is expressed as a percentage with $100 \%$ dose delivered at isocenter.

circular QA aperture without bolus. The increased collimation present in the case of the patient specific aperture resulted in greater interaction of the primary proton beam with the collimation material, leading to elevated doses outside the treatment field. Dose equivalent values of approximately 4 and $2 \mathrm{mSv} / \mathrm{Gy}$ were found at $2.5 \mathrm{~cm}$ from the edge of the primary beam for the patient specific aperture/bolus combination and the QA aperture, respectively. Sub-mSv readings were detected at distances greater than 10 and $20 \mathrm{~cm}$ from the field edge in the case of the QA aperture and patient specific combination, respectively. At a distance of $60 \mathrm{~cm}$ from the field edge the dose equivalent for the patient specific setup was $0.176 \mathrm{mSv} / \mathrm{Gy}$, which would result in a total dose equivalent at this point of $14 \mathrm{mSv}$ assuming a total dose delivery of 80 Gy to isocenter, which is the typical integral dose used in patient treatments.

Figure 6 shows results of measurements that were completed to assess the change in dose equivalent as a function of depth in a polystyrene phantom at $5 \mathrm{~cm}$ lateral distance from the patient specific proton field (series M1). For comparison, the central axis depth-dose distribution of the primary proton beam, as measured by a Markus chamber, is also shown and normalized to the dose at isocenter (100\%). Initial dose equivalent values at the surface of the phantom decreased by $38 \%$ after traversing $5 \mathrm{~cm}$ of phantom material.

Figure 7 displays the lateral distance dependence of dose equivalent at two different depths within the polystyrene phantom (measurement series M2). Measurements were completed at the plateau region and the level of the SOBP. The dose equivalent decreased with increasing distance from the field edge, similarly to what was measured at the phantom surface (Fig. 5), however with a more gradual falloff. Moreover, the falloff is less pronounced at the level of the SOBP compared to the shallower depth, which leads to a convergence of the two curves at a lateral displacement from the field edge of $20 \mathrm{~cm}$. This would indicate that secondaries produced inside the phantom contributed increasingly to the dose equivalent. 


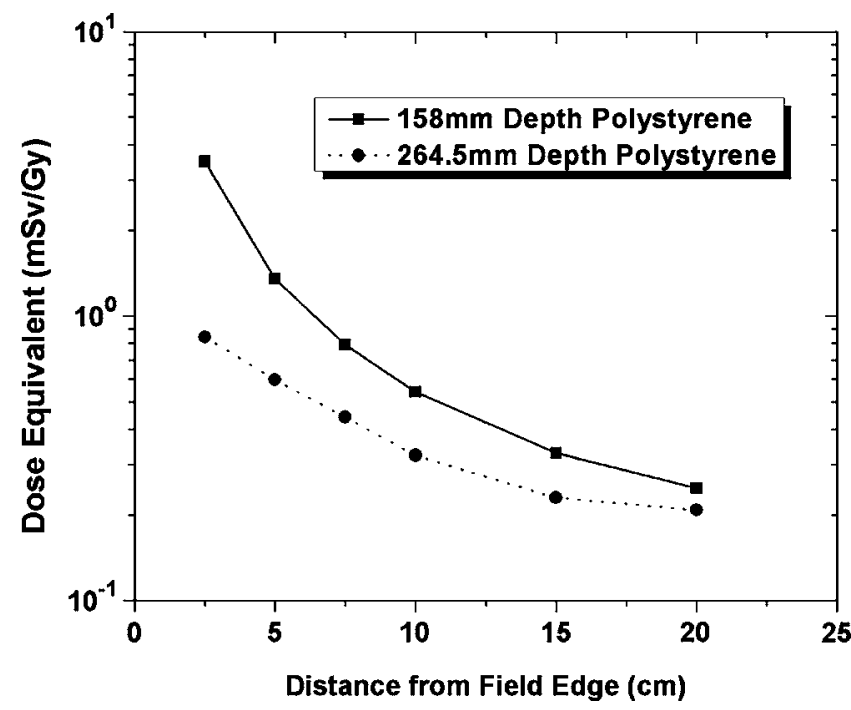

FIG. 7. Assessment of the dose equivalent as a function of lateral distance from the field edge at different depths within the polystyrene phantom.

Figure 8 displays the dose equivalent past distal edge of the SOBP in the polystyrene phantom (measurement series M3). The dose equivalent in the case of the typical prostate treatment investigated here was $1.6 \mathrm{mSv} / \mathrm{Gy}$ at $0.6 \mathrm{~cm}$ from the distal edge, and this value decreased exponentially with increasing distance from the distal edge. Fitting an exponential function to this trend resulted in the following relationship for the dose equivalent in $\mathrm{mSv}$ :

$$
H=1.65 e^{-0.1081 x},
$$

where $x$ is the distance in $\mathrm{cm}$ from the distal edge.

The average quality factor is valuable as it provides information on the types and abundances of radiation present. This information is presented in Fig. 9 as a function of lateral distance from the treatment field edge measured at the surface of the anthropomorphic phantom, past the distal edge of the SOBP in the homogeneous polystyrene phantom (mea-

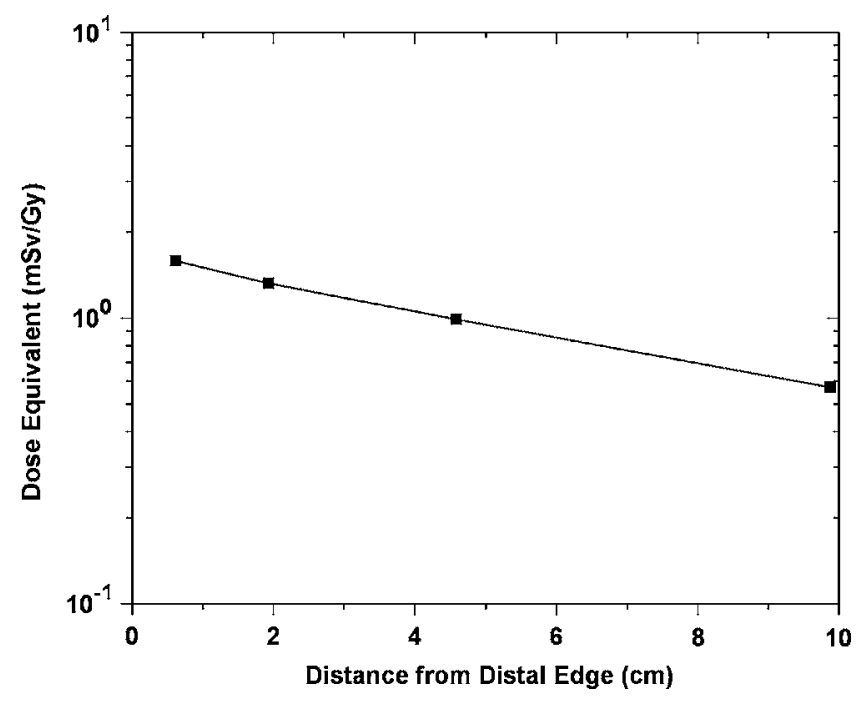

FIG. 8. Dose equivalent values past the distal edge of the SOBP. surement series M3), and also as a function of depth in polystyrene (measurement series M1). The average quality factor increased from 2 to 7 at a distance of 2.5 and $60 \mathrm{~cm}$ from the field edge, respectively. Figure 9(b) shows that the average quality factor past the SOBP remained relatively constant at an approximate value of 6.5. In this region, high energy primary protons were not present and the radiation field comprised of relatively low energy neutrons produced both from the beam-modifying/monitoring devices and within the phantom. Figure 9(c) indicates that the average quality factor does not change significantly in the proton plateau region, with a stable value of approximately 2.5 registered. At the level of the SOBP, the average quality factor increases to values of 5-6.

\section{DISCUSSION}

In this work, we have utilized a microdosimeter based on SOI technology to measure the depth and lateral distance dependence of the dose equivalent for a passively scattered proton beam used in a typical prostate cancer treatment. Unlike conventional TEPC devices, the SOI microdosimeter comprises an array of truly microscopic detection elements. This allows for faster data acquisition through the use of multiple SVs, good spatial resolution, and placement inside phantoms. The microdosimetry method also provides a more accurate estimation of dose equivalent using an established relationship between lineal energy and quality factor. This is particularly advantageous in mixed radiation field measurements.

SOI microdosimetry has certain limitations that need to be considered when evaluating the uncertainty of the measurements presented in this work. The lower level detection limit of the SOI device is approximately $0.8-1 \mathrm{keV} / \mu \mathrm{m}$, whereas the $y$ values in the $Q(y)$ distribution extend down to $0.1 \mathrm{keV} / \mu \mathrm{m}$. This limitation does not produce a significant error in the dose equivalent because the $Q$ value is less than 1 in this region. The main source of error in these measurements arises from the TE conversion factor, which is derived from the ratio of stopping powers. This factor covers a wide range of recoil proton energies from $0.1-200 \mathrm{MeV}$; hence, the value for each individual particle can lie from $0.5-0.8$ as shown in Fig. 10. Previous work with protons and alpha particles has found best agreement using a value of 0.63. By using this value, the systematic error introduced in the data presented here may be up to about $\pm 15 \%$. Additional work, including Monte Carlo simulations, is necessary to further reduce this uncertainty for proton therapy applications.

Measurements in polystyrene provided information on the change in dose equivalent (Figs. 6 and 7) and average quality factor (Fig. 9) as a function of depth and lateral displacement from the field edge. Figure 6 indicates that the initial dose equivalent values at the surface of the phantom decreased by $38 \%$ after traversing $5 \mathrm{~cm}$ of phantom material. This leads to the conclusion that many secondary particles produced within the passive scattering and collimation system do not penetrate to a great depth within the phantom. Such particles are probably thermal neutrons; however, Monte Carlo simu- 

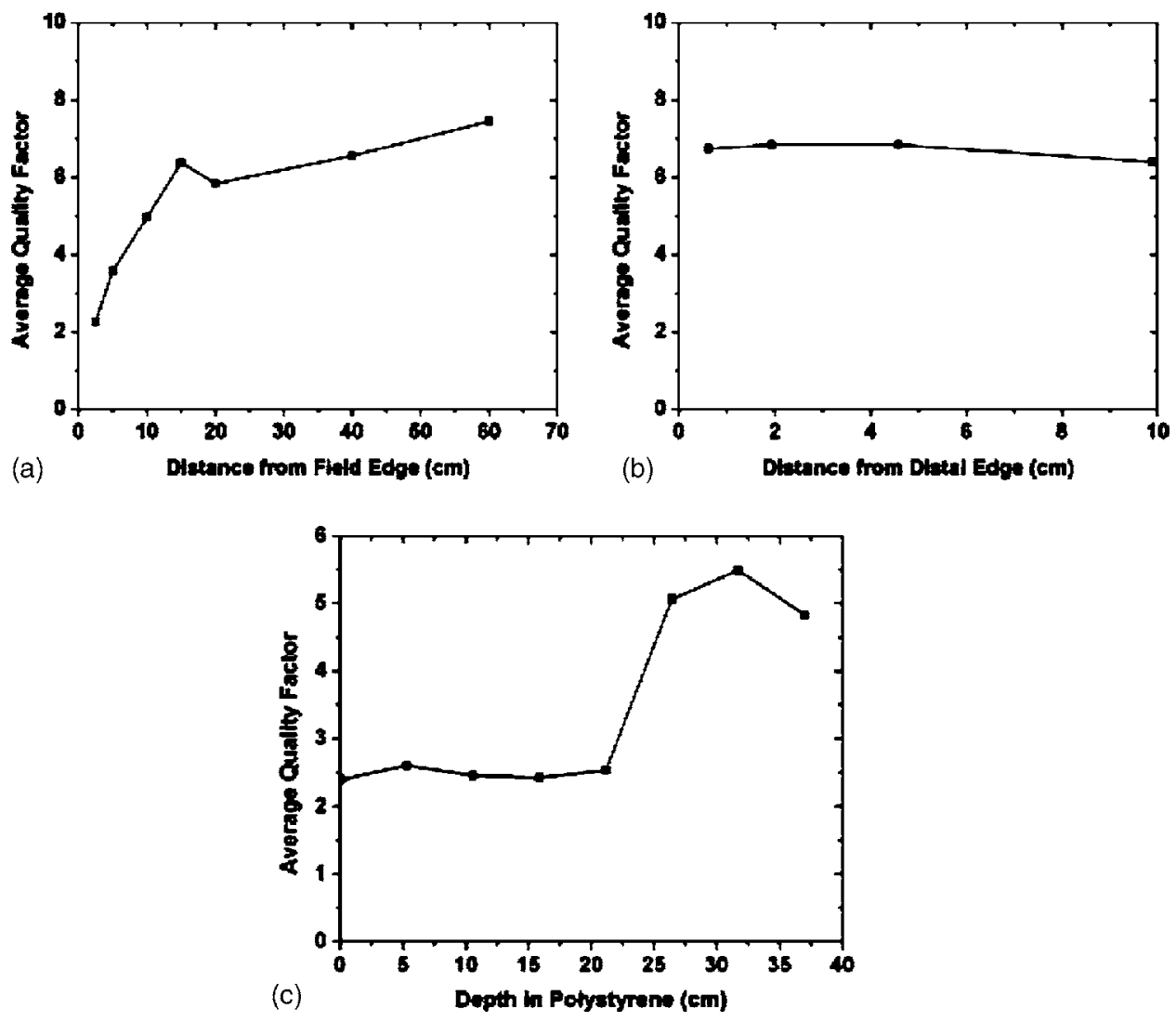

FIG. 9. Average quality factor values for (a) points lateral to the treatment field edge at the surface of the anthropomorphic phantom; (b) points past the SOBP within the polystyrene phantom; and (c) as a function of depth in polystyrene at $5 \mathrm{~cm}$ from the primary field edge. In each case, the aperture and bolus combination was that for a typical prostate patient.

lations would be needed to determine this with certainty. Figure 9(a) shows that at the surface of the phantom the quality factor increases with increasing distance from the lateral field edge. This indicates that, close to the field edge, scattered neutrons of higher energy and lower linear energy transfer (LET) made up a substantial portion of the dose equivalent, however, further from the field edge lower energy neutrons predominate resulting in higher average quality factors. In the region past the SOBP on the central beam axis the

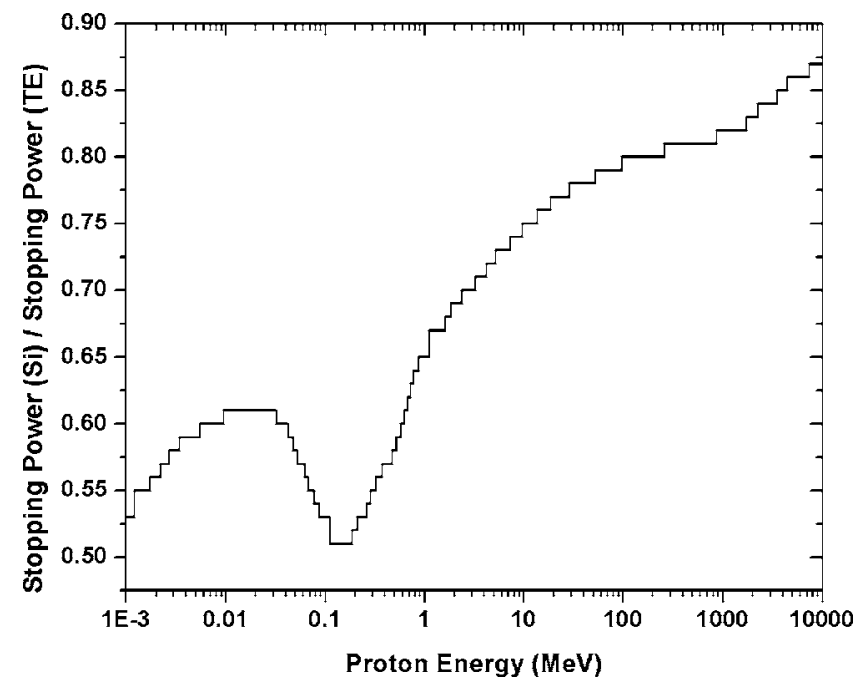

FIG. 10. Ratio of proton stopping powers for silicon and tissue (Ref. 25). The region of interest in this case was approximately $0.1-200 \mathrm{MeV}$. quality factor remains practically constant [Fig. 9(b)]. In this region, high energy primary protons are not present and the radiation field comprises of relatively low-energy neutrons produced both from the beam-modifying/monitoring devices and within the phantom. Figure 9(c) indicates that at a lateral distance of $5 \mathrm{~cm}$ from the field edge the average quality factor did not change significantly in the proton plateau region, with a stable value of approximately 2.5 registered while the dose equivalent decreases. At the level of the SOBP, the average quality factor increased to values of 5-6 indicating the presence of higher LET particles, which are predominantly slowing protons.

The SOI results presented here provide an estimate of the dose equivalent both at the surface and at depth that a prostate cancer patient will experience during proton therapy. They show that dose equivalent values are of the order of a few $\mathrm{mSv} / \mathrm{Gy}$ within $10 \mathrm{~cm}$ of the treatment volume (Figs. 5 and 7) and appear to level off at about $0.2 \mathrm{mSv}$ for larger distances from the treatment volume (Fig. 7). These dose equivalent values compare favorably to those experienced by patients from IMRT treatments ${ }^{2}$ and cone beam CT imaging that is increasingly used for image guided radiation therapy. ${ }^{26}$

It is important when applying existing technology to a new mode of measurement to benchmark results against existing data in this field. Figure 11 illustrates the comparison of results from other centers using different measurement techniques. At the Midwest Proton Radiotherapy Institute (MPRI), neutron doses were measured with a neutron bubble 


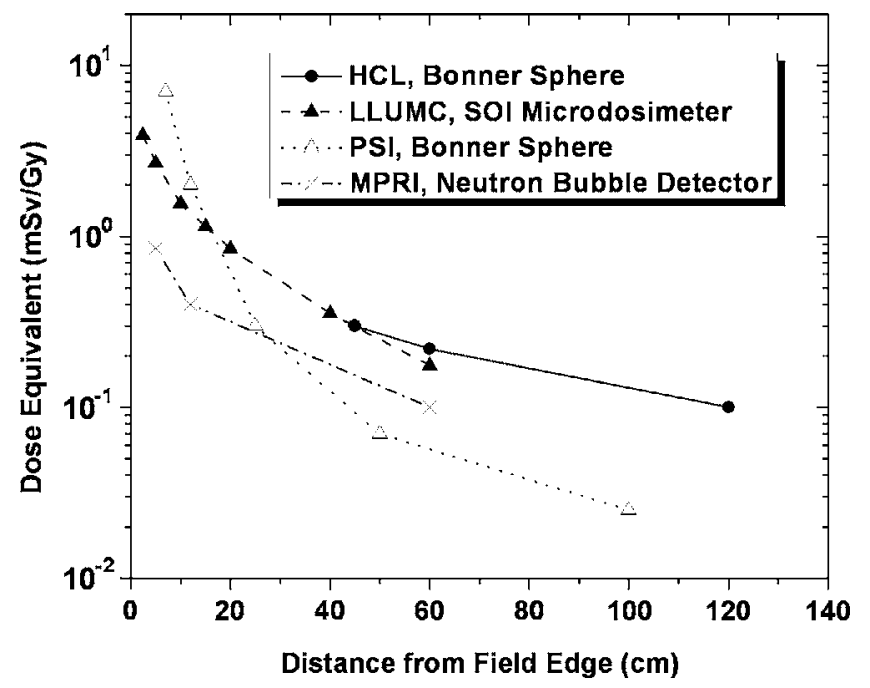

FIG. 11. Comparison of dose equivalent values determined using different methods. In this figure results are presented from HCL (Ref. 11), PSI (Ref. 13), and MPRI (Ref. 12) in comparison with results presented in this paper using the SOI microdosimeter and the patient specific aperture/bolus combination (Fig. 5).

detector and a passively scattered and modulated proton beam of $12 \mathrm{~cm}$ range in water. The field size in this instance was $10 \mathrm{~cm}$ in diameter with an $8 \mathrm{~cm}$ modulation delivered to the midline of an anthropomorphic phantom. Measurements were made as a function of distance from the proton field edge within the phantom, with the detector situated within the abdomen to determine the scattered neutron dose at the location of a fetus. Measurements were completed for two snout sizes $(10$ and $20 \mathrm{~cm}$ ), with the $20 \mathrm{~cm}$ results presented here. The Harvard Cyclotron Laboratory (HCL) data were derived on the $160 \mathrm{MeV}$ beamline used for patient treatments utilizing Bonner spheres for the detection of neutrons. A Lucite phantom $(26 \mathrm{~cm}$ in diameter and $24 \mathrm{~cm}$ in length) represented the patient, while the primary proton radiation field had a cross-sectional area of $5 \times 5 \mathrm{~cm}^{2}$ and a range of $16 \mathrm{~cm}$ with a modulation of $8.2 \mathrm{~cm}$. The neutron dose equivalent per unit proton absorbed dose was measured as a function of vertical distance below isocenter. Finally, the data from the Paul Scherrer Institute (PSI) provide information on the secondary neutron dose delivered during a spot-scanning treatment. Again, this study utilized Bonner spheres as the radiation detection apparatus with an incident beam of $177 \mathrm{MeV}$ and no range shifter plates in the beam path. A water phantom with $0.5 \mathrm{~cm}$ thick Lucite walls was used to simulate the patient. The values of effective neutron dose were measured laterally from the water phantom at the level of the treatment volume, not the surface of the phantom as in the case presented here.

Despite the difference in experimental conditions and measurement techniques, the agreement between the data is good. Of note, the equivalent doses measured with the spotscanning beam were consistently lower than the other data, in particular at distances greater than $50 \mathrm{~cm}$. This is consistent with the fact that the spot-scanning system does not contain beam modifying devices such as apertures and scat- tering foils which contribute neutrons to the radiation field outside the primary treatment field. The results from PSI, HCL, and MPRI consisted of lower incident proton energies than the measurements completed with the SOI microdosimeter, which may have a bearing on the results as lower incident proton energies have in turn lower cross sections for inelastic reaction.

\section{CONCLUSION}

This study has shown the applicability of the SOI microdosimeter in determining the dose equivalent outside proton treatment fields, and has provided valuable information of the dose equivalent both at the surface and at depth experienced by prostate cancer patients treated with protons. Further studies utilizing the SOI microdosimeter should be conducted with therapeutic proton fields generated with both passive and active beam delivery techniques under otherwise equivalent conditions and for different clinical treatment scenarios including pediatric cancers. It will also be useful in such studies to compare the response of SOI detector to other microdosimetric and neutron measurement techniques.

\section{ACKNOWLEDGMENTS}

The first author of this paper would like to acknowledge the support received from the Australian American Fulbright Association. The authors would also like to acknowledge the support of the Australian Institute of Nuclear Science and Engineering (AINSE) and the Australian Nuclear Science and Technology Organization (ANSTO) in benchmark testing of the SOI microdosimeter. Further, the authors would like to acknowledge support received from the staff from the Department of Radiation Medicine at Loma Linda University Medical Center, who helped make this work possible.

\footnotetext{
${ }^{a)}$ Electronic mail: ajw16@uow.edu.au

${ }^{1}$ American Cancer Society Statistics for 2004, http://www.cancer.org/ docroot/stt/stt_0.asp (2004).

${ }^{2}$ E. J. Hall, "Intensity-modulated radiation therapy, protons, and the risk of second cancers," Int. J. Radiat. Oncol. Biol. Phys. 65, 1-7 (2006).

${ }^{3}$ J. D. Slater, "Clinical applications of proton radiation treatment at Loma Linda University: Review of a fifteen-year experience," Technol. Cancer Res. Treat. 5, 81-89 (2006).

${ }^{4}$ J. D. Slater et al., "Proton therapy for prostate cancer: The initial Loma Linda University experience," Int. J. Radiat. Oncol. Biol. Phys. 59, $348-$ 352 (2004).

${ }^{5}$ A. M. Koehler, R. J. Schneider, and J. M. Sisterson, "Flattening of proton dose distributions for large-field radiotherapy," Med. Phys. 4, 297-301 (1977).

${ }^{6}$ R. R. Wilson, "Radiological use of fast protons," Radiology 47, 487-491 (1946).

${ }^{7}$ A. Lomax et al., "Intensity Modulated Proton Therapy at PSI: Things we have learnt (and are still learning)," Radiother. Oncol. 76, S54-S55 (2005).

${ }^{8}$ A. J. Lomax et al., "Spot scanning proton therapy: Treatment planning and treatment verification," Radiother. Oncol. 78, S21-S21 (2006).

${ }^{9}$ E. Pedroni et al., "Initial experience of using an active beam delivery technique at PSI," Strahlenther. Onkol. 175, 18-20 (1999).

${ }^{10}$ E. Pedroni, "The new proton scanning gantry of PSI: A system designed for IMPT delivery in the whole body including moving targets," Radiother. Oncol. 78, S71-S71 (2006).

${ }^{11} \mathrm{X}$. Yan et al., "Measurement of neutron dose equivalent to proton therapy patients outside of the proton radiation field," Nucl. Instrum. Methods Phys. Res. A 476, 429-434 (2002).
} 
${ }^{12} \mathrm{G}$. Mesoloras et al., "Neutron scattered dose equivalent to a fetus from proton radiotherapy of the mother," Med. Phys. 33, 2479-2490 (2006).

${ }^{13} \mathrm{U}$. Schneider et al., "Secondary neutron dose during proton therapy using spot scanning," Int. J. Radiat. Oncol. Biol. Phys. 53, 244-251 (2002).

${ }^{14}$ ICRU 1983 Microdosimetry Report 36, Washington, DC, 1983.

${ }^{15} \mathrm{H}$. H. Rossi and M. Zaider, Microdosimetry and Its Applications (Springer, London, 1996).

${ }^{16}$ P. D. Bradley, A. B. Rosenfeld, and M. Zaider, "Solid state microdosimetry,” Nucl. Instrum. Methods Phys. Res. B 184, 135-157 (2001).

${ }^{17}$ A. B. Rosenfeld et al., "Solid state microdosimetry in hadron therapy," Radiat. Prot. Dosimetry 101, 431-434 (2002).

${ }^{18}$ A. Rosenfeld et al., "Method of Monte Carlo simulation verification in hadron therapy with non-tissue equivalent detectors," Radiat. Prot. Dosimetry 119, 487-490 (2006).

${ }^{19}$ A. B. Rosenfeld et al., "A new silicon detector for microdosimetry applications in proton therapy,” IEEE Trans. Nucl. Sci. 47, 1386-1394 (2000).
${ }^{20}$ P. D. Bradley et al., "Performance of silicon microdosimetry detectors in boron neutron capture therapy," Radiat. Res. 151, 235-243 (1999).

${ }^{21} \mathrm{~A}$. Wroe et al., "Silicon microdosimetry in heterogeneous materials: Simulation and experiment," IEEE Trans. Nucl. Sci. 53, 3738-3744 (2006).

${ }^{22}$ International Commission on Radiation Units and Measurements, "The quality factor in radiation protection," Report ICRU-40 (1986).

${ }^{23}$ I. Cornelius et al., "Ion beam induced charge characterisation of a silicon microdosimeter using a heavy ion microprobe," Nucl. Instrum. Methods Phys. Res. B 190, 335-338 (2002).

${ }^{24}$ NIST, National Institute of Standards and Technology Composition Database Program, http://physics.nist.gov/cgi-bin/Star/compos.pl?ap

${ }^{25}$ NIST, National Institute of Standards and Technology PSTAR Database Program, http://physics.nist.gov/PhysRefData/Star/Text/PSTAR.html

${ }^{26} \mathrm{D}$. Letourneau et al., "Cone-beam-CT guided radiation therapy: Technical implementation,” Radiother. Oncol. 75, 279-286 (2005). 